\title{
Resveratrol Combined with Pioglitazone Ameliorates Cardiovascular Complications in db/db Diabetic Mice
}

Miguel Ángel Duarte-Vázquez ${ }^{1}$, Antonieta Gomez Solis ${ }^{2}$, Jorge Reyes Esparza ${ }^{2}$, Jorge Luis Rosado ${ }^{3}$ and Lourdes Rodríguez Fragoso $^{2}$

${ }^{1}$ Department of Research and Development, Nucitec, S.A. de C.V., Querétaro, Querétaro, Mexico

${ }^{2}$ Universidad Autónoma del Estado de Morelos, Cuernavaca, Morelos, Mexico

${ }^{3}$ Facultad de Ciencias Naturales, Universidad Autónoma de Querétaro, Querétaro, Querétaro, México

"Corresponding author: Lourdes Rodriguez Fragoso, Facultad de Farmacia, Universidad Autónoma del Estado de Morelos, Chamilpa 1001, Cuernavaca, Morelos, Mexico, Tel: 0152777 329-7000; Fax: 828-299-5980; E-mail: mrodriguezf@uaem.mx

Received date: Nov 23, 2018; Accepted date: Nov 27, 2018; Published date: Dec 04, 2018

Copyright: (C) 2018 Duarte-Vázquez MA, et al. This is an open-access article distributed under the terms of the Creative Commons Attribution License, which permits unrestricted use, distribution, and reproduction in any medium, provided the original author and source are credited.

\begin{abstract}
Type 2 Diabetes Mellitus patients are predisposed to serious cardiovascular morbidity and mortality. Diabetes causes different structural and functional alterations in the myocardial tissue, which are induced by hyperglycemia, insulin resistance, and hyperlipidemia. The resulting cardiomyopathy is characterized by myocardial fibrosis, dysfunctional remodeling and eventually, clinical heart failure. This study addressed the effects of resveratrol and pioglitazone to ameliorate diabetic cardiovascular complications using a $\mathrm{db} / \mathrm{db}$ diabetic mice model. Male $\mathrm{db} / \mathrm{db}$ diabetic mice were randomly assigned to orally receive resveratrol $(50 \mathrm{mg} / \mathrm{kg}, \mathrm{n}=5)$, resveratrol/pioglitazone $(50$ $\mathrm{mg} / \mathrm{kg}$ and $20 \mathrm{mg} /$ day, respectively, $\mathrm{n}=5)$ for the space of 6 weeks. Non-diabetic lean mice $(\mathrm{n}=5)$ were included as a control group. Histopathological analyses were performed in heart and aortic vessel tissue samples using hematoxylin and eosin, as well as Masson Trichromic staining. VCAM-1 immunohistochemistry was also assessed. Blood glucose, hemoglobin A1c, insulin, glycosuria, triglycerides, cholesterol, and LDL cholesterol were all examined. Present data suggest that the combination of pioglitazone plus resveratrol significantly lower circulating levels of insulin, hemoglobin A1c, glucose, triglycerides, cholesterol, and LDL levels. Our results also show that tissue damage to the heart and aortic vessels caused by diabetes can also be improved by the administration of said combination. The present study demonstrates that resveratrol/pioglitazone combination therapy improves carbohydrate and lipid metabolism, as well as improving diabetic cardiomyopathy in diabetic mice by producing a synergistic pharmacological effect. The combination of resveratrol plus pioglitazone has therapeutic potential against diabetic cardiomyopathy.
\end{abstract}

Keywords: Resveratrol; Pioglitazone; Cardiovascular; Diabetes mellitus; Glucose; Triglycerides; Cardiomyopathy; Haemoglobin A1c

\section{Introduction}

Patients suffering from Type 2 Diabetes Mellitus (DM) are more prone to develop cardiovascular complications [1]. The cardiovascular adaptations present in Type 2 DM (i.e. atherosclerosis, hypertension, ischemic disease, stroke, etc.) are associated with diverse molecular and cellular responses mainly derived from insulin resistance, an excess of free fatty acids, hyperglycemia, inflammation, thrombosis, and hypertension $[2,3]$. Cardiac insulin resistance is characterized by a cellular and tissue reorganization leading to cardiomyopathy, cardiac fibrosis and impaired molecular signaling, which in turn cause the death of myocytes and endothelial cells [4]. The mechanisms involved in the progression of such cardiovascular disease include mainly chronic inflammation, cytokine imbalance, glucotoxicity and oxidative stress $[5,6]$.

Current pharmacological therapies for diabetic cardiomyopathy are focused on regulating the glucose metabolism, oxidative damage, calcium handling, carbohydrate utilization in cardiomyocytes, and the preservation of the myocardium's structure and function [7-13]. Pioglitazone is a glucose-lowering agent that protects against cardiovascular disease associated with Type 2 DM. Pioglitazone reduces the most important cardiovascular events in Type $2 \mathrm{DM}$ patients because it improves cardiac insulin resistance, carbohydrate and lipid metabolism, and reduces inflammation $[14,15]$.

Resveratrol appears to have a promising protective effect on cardiovascular disorders related to Type 2 DM. During the past decade, increasing evidence supports the beneficial effects of resveratrol on several chronic diseases and its validity has been challenged in several studies, including Type 2 DM [16]. Some of the potential effects reported for resveratrol include an improvement of lipid metabolism, glucose homeostasis and mitochondrial biogenesis, reduced inflammation, an increase in antioxidant defences, a reduction of Reactive Oxygen Species (ROS), and an improvement on atherosclerosis [17-19]. Diabetic cardiomyopathy is globally on the rise, given its frequently asymptomatic early stages; there is a need for research on new therapeutic strategies that take advantage of the important protective effects of both pioglitazone and resveratrol.

This study addressed the effects of resveratrol, alone or combined with conventional pioglitazone, on cardiovascular complications induced by diabetes using a $\mathrm{db} / \mathrm{db}$ diabetic mice model.

\section{Material and Methods}

\section{Animals}

Male $\mathrm{db} / \mathrm{db}$ diabetic mice (BKS.Cg-+ Leprdb/+Leprdb/OlaHsd) and male lean mice (BKS.Cg-Lean/OlaHsd) were obtained at 8 weeks of 
age from Envigo SA de CV (Mexico, Mexico). All animals were kept in a temperature and humidity-controlled location and were allowed to have food and water ad libitum.

The protocol was approved by the Autonomous University of Querétaro Committee and was conducted in accordance with the international guides for experimental animal handling [20].

\section{Pharmacological treatments}

Diabetic animals were split into three groups, each one consisting of 5 animals. Non-diabetic lean mice were included as a control group $(n=5)$. The diabetic mice were randomly assigned to one of the following groups: one group received $200 \mu \mathrm{L}$ of water orally; a second group received resveratrol monotherapy with a daily dose of $50 \mathrm{mg} / \mathrm{Kg}$ body weight p.o. in $200 \mu \mathrm{L}$ of water, and the third group received a combined resveratrol/pioglitazone treatment with a daily dose of 50 $\mathrm{mg} / \mathrm{Kg}$ and $20 \mathrm{mg} /$ day p.o, respectively.

During treatment, all groups received a controlled food and water ad libitum. All treatments were administered for 6 weeks. Pioglitazone was purchased from Wan bury Limited (Maharashtra, India), and resveratrol $\left(\right.$ resVida ${ }^{\circ}$ ) was provided by DSM Nutritional Products (Mexico, Mexico). Both drugs were dissolved in sterile deionized water prior to treating animals. Mice body weight was monitored throughout the study. At the end of treatment, animals were sacrificed under light anesthesia.

Blood and tissue samples (heart and aortic vessels) were collected from each animal and kept at $-4^{\circ} \mathrm{C}$ until further studies.

\section{Biochemical analysis}

All the biochemical parameters were quantified at the end of treatment (6 weeks), blood samples were obtained from cardiac puncture. Glucose, triglycerides, cholesterol, Haemoglobin A1c, and insulin levels were quantified using colorimetric methods (Triglycerides SL, Cholesterol PAP SL, Glucose PAP SL, ELITech, Mexico, anti-Haemoglobin AIc antibody Abcam kit and rat/mouse insulin ELISA kit from Millipore, USA) in serum in accordance with manufacturer protocol.

Weekly monitoring of blood glucose levels was done after obtaining the blood samples through the vein of the tail.

\section{Histopathological analysis}

Tissue fragments of heart and aortic vessels were fixed in $10 \%$ formaldehyde solution, dissolved in phosphate-saline buffer ( $\mathrm{pH} 7.4)$, dehydrated in alcohol and embedded in paraffin. Four-micrometer paraffin tissue heart sections were stained with hematoxylin and eosin ( $\mathrm{H}$ and $\mathrm{E}$ ) and Masson Trichrome staining, and then subjected to histopathological examination. Aortic vessels were stained with $\mathrm{H}$ and E stain. Rabbit anti mice VCAM 1 polyclonal antibody (1:300, bs 0920R; Beijing Biosynthesis Biotechnology Co., Ltd., Beijing, China) was used to incubate the heart sections at $4^{\circ} \mathrm{C}$ overnight.

Horseradish peroxidase labeled goat anti-rabbit immunoglobulin $\mathrm{G}$ was used for incubation for $30 \mathrm{~min}$ at room temperature. Peroxidase diaminobenzidine reaction and Masson staining were subsequently performed. The sections were sealed with resin and examined under a microscope.

For the quantification of fibrosis and VCAM-1 twenty high power fields were randomly selected from each slice and photographed at 40X magnification and then analyzed employing the Image-Pro Insight 9 software (Media Cybernetics Inc.). Results were expressed as percent of control. Macroscopic observations of the heart and the aorta were also done.

\section{Statistical analysis}

All statistical analyses were analyzed using SPSS 10.0 software (SPSS Inc., Chicago, Ill, USA). For blood glucose measurements was used two ways repeated measurements ANOVA. Statistical significance for the biochemical analyses, fibrosis and VCAM-1 determination was calculated using ANOVA, as well as Turkey's test for multiple comparatives.

Results are expressed as mean \pm SEM. Differences were considered significant if the $\mathrm{p}$-value was less than $0.05(\mathrm{p}<0.05)$.

\section{Results}

\section{Glucose metabolism}

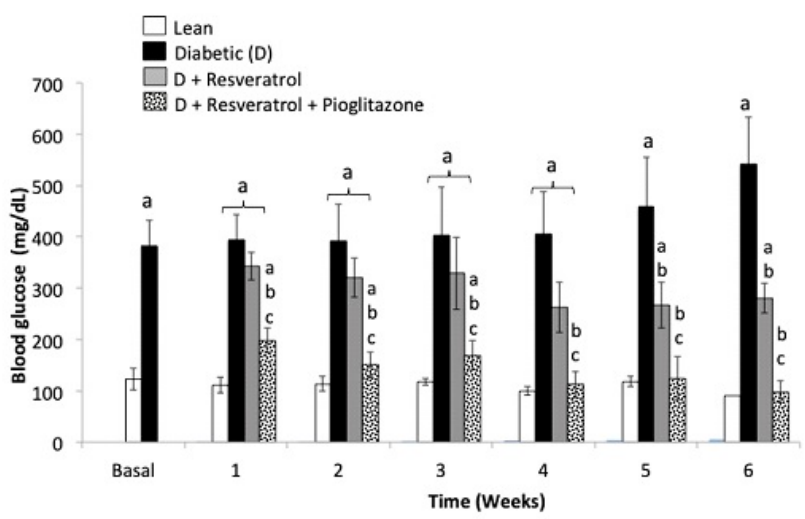

Figure 1: Variations in blood glucose levels in male $\mathrm{db} / \mathrm{db}$ diabetic mice over 6 weeks. Each bar represents the mean \pm SEM. All groups consisted of five animals. a means different from lean mice $(p<0.05)$; b means different from diabetic mice $(p<0.05)$, and $c$ means different from diabetic mice treated with resveratrol $(\mathrm{p}<0.05)$.

Figure 1 shows the blood glucose levels observed in animals during the time they received the treatments. Lean mice kept blood glucose levels between 93 to $112 \mathrm{mg} / \mathrm{dL}$ during 6 weeks, while diabetic mice started with levels near to $400 \mathrm{mg} / \mathrm{dL}$ and these increased over the next weeks $(\mathrm{p}<0.05)$; at the end, their levels were around $550 \mathrm{mg} / \mathrm{dL}$ $(\mathrm{p}<0.05)$.

Diabetic mice treated with Resveratrol $(50 \mathrm{mg} / \mathrm{Kg}$ ) alone had blood glucose levels of $342 \mathrm{mg} / \mathrm{dL}$ during the first week, but these were slightly reduced until they attained levels of $280 \mathrm{mg} / \mathrm{dL}$ on the sixth week of treatment $(\mathrm{p}<0.05)$. Diabetic mice treated with Resveratrol and Pioglitazone showed a significant reduction in blood glucose levels since the first week $(198 \mathrm{mg} / \mathrm{dL})$, and their levels decreased significantly, reaching levels similar to those of lean mice at 6 weeks of treatment $(98.2 \mathrm{mg} / \mathrm{dL}, \mathrm{p}<0.05)$. 
Observed blood glucose levels at the end of 6 weeks correlated with a reduction in levels of hemoglobin A1C and insulin, as well as with a significant reduction in glycosuria (Table 1). Haemoglobin A1c levels doubled in diabetic mice when compared with lean mice $(\mathrm{p}<0.05)$. Resveratrol treatment alone did not show significant changes when compared with diabetic mice. However, diabetic mice treated with Resveratrol and Pioglitazone showed a significant reduction in Haemoglobin A1c level $(\mathrm{p}<0.05)$. Insulin levels showed a significant increase in diabetic mice as well as those treated with Resveratrol alone.
However, animals treated with Resveratrol and Pioglitazone showed an important reduction in insulin levels $(\mathrm{p}<0.05)$. Glycosuria also showed a significant increase in diabetic mice; however, diabetic mice treated with Resveratrol alone and those treated with the Pioglitazone combination showed values quite similar to those of lean mice $(\mathrm{p}<0.05)$.

It is important to mention that animals in each group consumed the same amount of food (data not shown) and there were no differences between diabetic mice and those animals treated with resveratrol alone and combined with pioglitazone (Table 1).

\begin{tabular}{|l|l|l|l|l|}
\hline & Lean & Diabetic & Diabetic Resveratrol & Diabetic Resveratrol+Pioglitazone \\
\hline Glucose $(\mathrm{mg} / \mathrm{dL})$ & $93.33 \pm 7.7$ & $541.6 \pm 92 \mathrm{a}$ & $280.7 \pm 29 \mathrm{ab}$ & $98.2 \pm 20.8 \mathrm{bc}$ \\
\hline Hemoglobin $\mathrm{A} 1 \mathrm{c}(\%)$ & $2.2 \pm 0.3$ & $4.6 \pm 0.4 \mathrm{a}$ & $4.3 \pm 0.4 \mathrm{a}$ & $3.2 \pm 0.1 \mathrm{abc}$ \\
\hline Insulin $(\mathrm{ng} / \mathrm{mL})$ & $1.09 \pm 0.2$ & $12.9 \pm 2.5 \mathrm{a}$ & $9.6 \pm 2.2 \mathrm{a}$ & $3.6 \pm 0.6 \mathrm{a}$ \\
\hline Glycosuria $(\mathrm{mg} / \mathrm{dL})$ & $160.3 \pm 30$ & $809.4 \pm 96 \mathrm{a}$ & $151.7 \pm 59 \mathrm{ab}$ & $100 \pm 17 \mathrm{ab}$ \\
\hline Weight gain $(\mathrm{g})$ & $4 \pm 0.3$ & $7.13 \pm 2.73 \mathrm{a}$ & $9.28 \pm 1.48 \mathrm{a}$ & $10.25 \pm 2.58 \mathrm{a}$ \\
\hline $\begin{array}{l}\text { (a) } \mathrm{p}<0.05 \text { as compared with lean group } \\
\text { b) } \mathrm{p}<0.05 \text { as compared with diabetic group }\end{array}$ & & & \\
c) $<0.05$ as compared with resveratrol group & & & \\
\hline
\end{tabular}

Table 1: Alterations in glucose metabolism and weight gain after 6 weeks of treatment.

\section{Lipid profile}

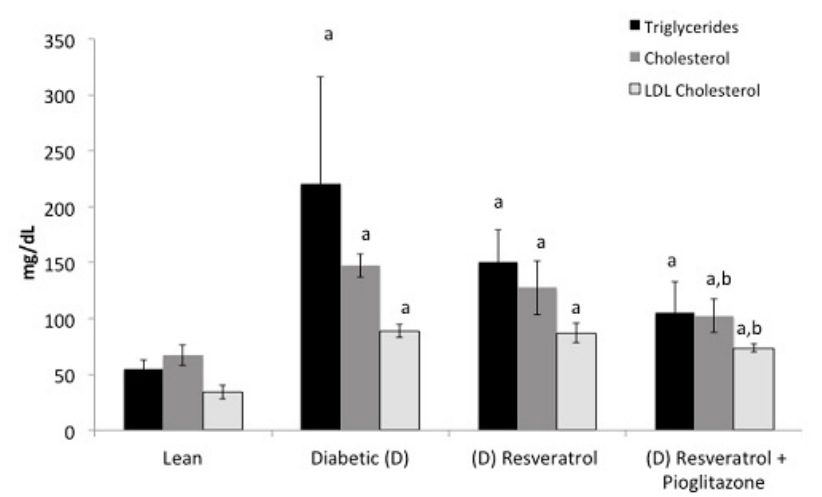

Figure 2: Profile of blood lipids in male $\mathrm{db} / \mathrm{db}$ diabetic mice. Each bar represents the mean \pm SEM. Animals were treated for 6 weeks. All groups consisted of five animals; a means different from lean mice $(\mathrm{p}<0.05)$; $\mathrm{b}$ means different from diabetic mice $(\mathrm{p}<0.05)$, and $\mathrm{c}$ means different from diabetic mice treated with resveratrol $(\mathrm{p}<0.05)$.

Figure 2 shows the lipid profile observed in all treated groups. As we can see, only diabetic mice treated with Resveratrol and Pioglitazone in combination showed a significant reduction in triglycerides, cholesterol and LDL levels $(\mathrm{p}<0.05)$ when compared with diabetic mice. Resveratrol alone was not able to significantly modify the lipid profile. Figure 3 shows the macroscopic characteristics of cardiovascular tissue observed in animals affected by the different treatments. Diabetic mice showed abundant adipose tissue surrounding the heart and thoracic aorta.

Animals treated exclusively with resveratrol had abundant adipose tissue in the thoracic aorta, but not the heart. However, diabetic mice treated with resveratrol and pioglitazone a reduction of adipose tissue in cardiovascular tissue was observed

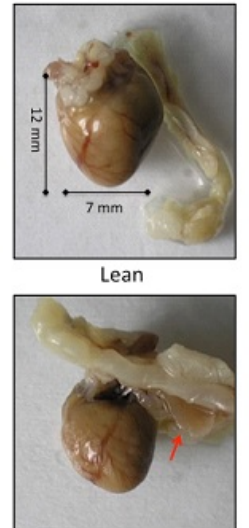

(D) Resveratrol

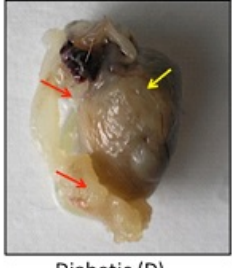

Diabetic (D)

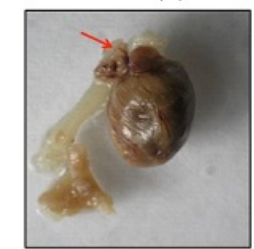

(D) Resveratrol + Pioglitazone
Figure 3: Macroscopic characteristics of cardiovascular tissue in male $\mathrm{db} / \mathrm{db}$ diabetic mice after six weeks of treatment. The red arrow indicates the presence of fat surrounding the thoracic aortas. The yellow arrow indicates the presence of fat surrounding the myocardium. 
Citation: Duarte-Vazquez MA, Solis AG, Esparza JR, Rosado JL, Fragoso LR (2018) Resveratrol Combined with Pioglitazone Ameliorates Cardiovascular Complications in db/db Diabetic Mice. J Metabolic Synd 7: 243. doi:10.4172/2167-0943.1000243

Page 4 of 7

\section{Histopathological results}

In order to measure the degree of damage in the myocardial tissue of diabetic mice, we employed Masson's trichrome stain to visualize the presence of fibrosis and cardiac remodeling (Figure 4).

Cardiac tissue from diabetic mice showed the presence of abundant connective tissue (fibrosis) surrounding the ventricular space and between myocardiocytes (18-fold, as compared with the lean group, $\mathrm{p}<0.05)$.

We also observed degenerative changes in myocardial cells. However, diabetic mice treated with Resveratrol combined with Pioglitazone showed a significant reduction of fibrotic tissue $(p<0.05)$ and the morphological aspect of their tissue was quite similar to that of lean mice.

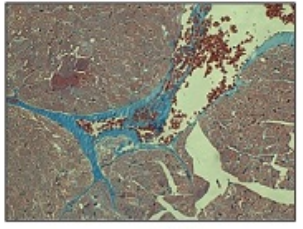

Lean

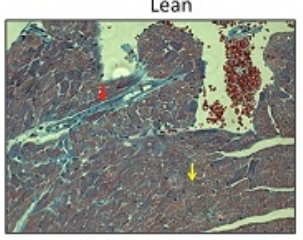

(D) Resveratrol

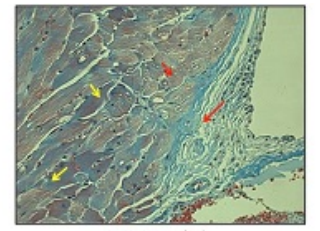

Diabetic (D)

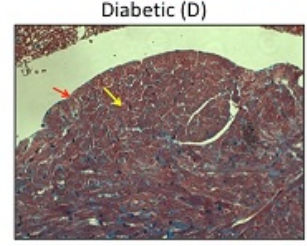

(D) Resveratrol + Pioglitazone

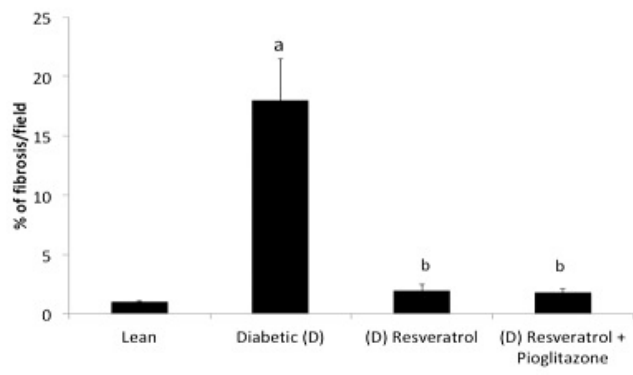

Figure 4: Histological examinations of cardiac tissue sections from male $\mathrm{db} / \mathrm{db}$ diabetic mice. Cardiac tissues were examined using Masson's trichrome staining: fibrosis can be recognized by blue staining into the myocardium (40X). Each bar represents the mean \pm SEM. All groups consisted of five animals. A means different from lean mice $(p<0.05)$; $b$ means different from diabetic mice $(p<0.05)$, and $c$ means different from diabetic mice treated with resveratrol $(\mathrm{p}<0.05)$.

We quantified and analyzed VCAM-1 as a marker molecule of the chronic vascular inflammatory process, key in the development of atherosclerotic disease.

Figure 5 shows the expression of VCAM-1 in myocardial tissue from diabetic mice that received treatment. There was an important increase in the expression of VCAM-1 in diabetic mice (12-fold) $(\mathrm{p}<0.05)$.

The distribution of VCAM-1 mainly surrounded the ventricles, vessels, and myocardial areas. However, diabetic mice treated with
Resveratrol combined with Pioglitazone showed a significant reduction in VCAM-1 expression when compared with diabetic mice $(\mathrm{p}<0.05)$. Expression was limited to vascular endothelium, as was the case in lean mice.

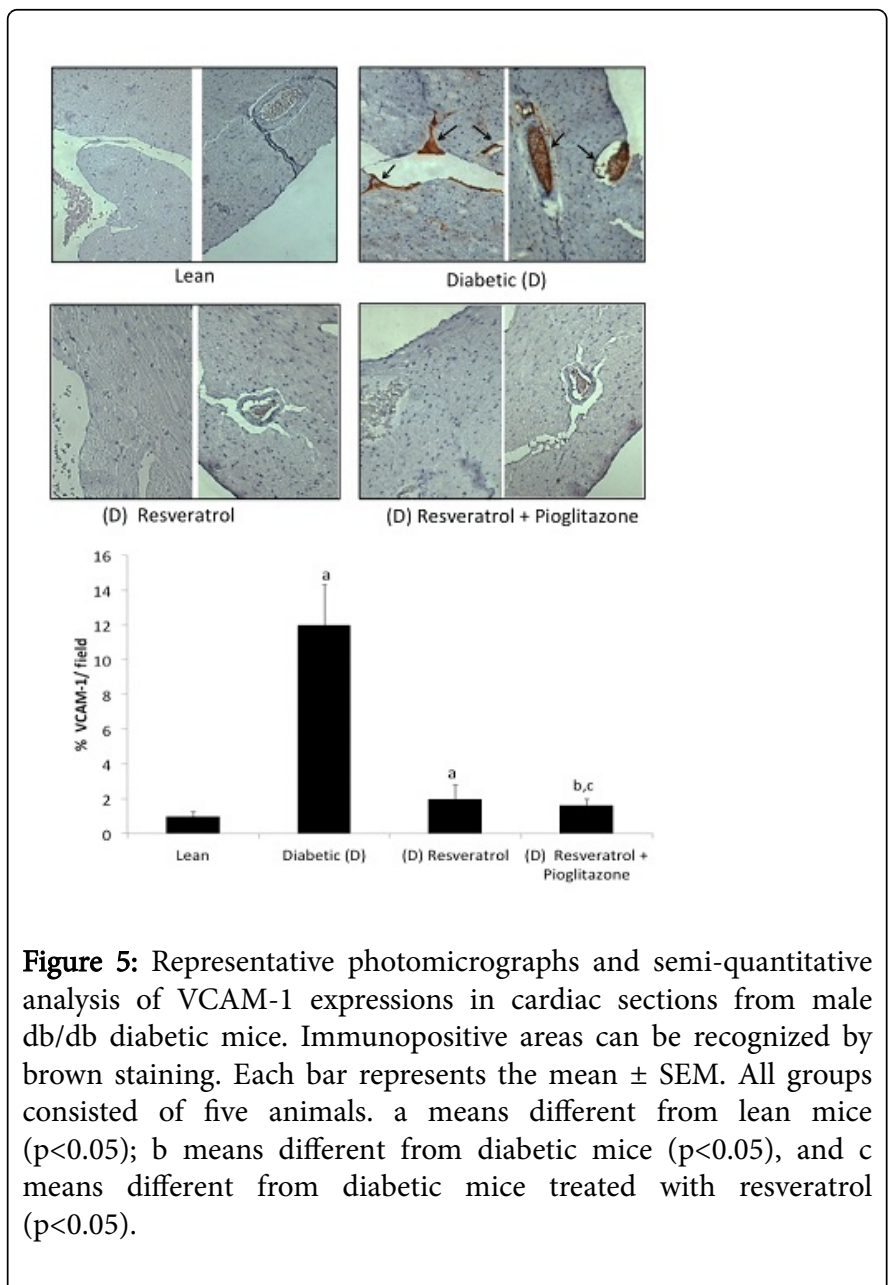

Figure 6 shows the characteristics of the thoracic aorta and cardiac arteries. Thoracic aorta from diabetic mice showed the presence of atherosclerotic plaques.

The presence of perivascular adipose tissue was evident in the wall, as was fat infiltration in the tunica media and a thickening of the wall of the aorta. Cardiac arteries showed a thickening of the vascular wall, perivascular fibrosis, and the presence of atherosclerotic plaques in the vessels' Lumina.

Animals treated with Resveratrol combined with Pioglitazone showed aortas, cardiac arteries and myocardial tissue with morphological characteristics similar to those of lean mice.

Black arrows indicate adipose tissue and fat deposits; black bars indicate the thickness of the vascular wall; asterisks indicate atheroma. Cardiac tissue sections were examined using Masson's trichrome staining: fibrosis can be recognized by blue staining. The thickness of the vascular wall is different depending on the treatment (40X). 


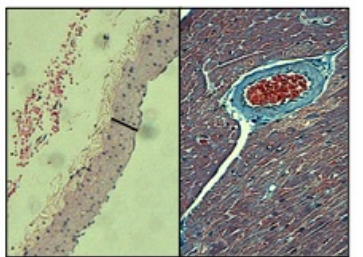
Lean

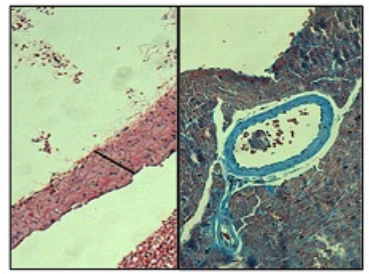

(D) Resveratrol
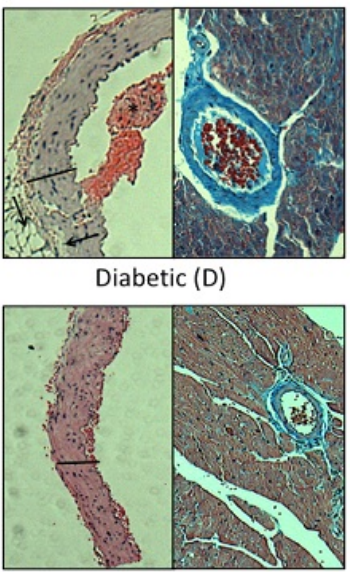

(D) Resveratrol + Pioglitazone
Figure 6: Representative photomicrographs of aortas and cardiac artery sections from male $\mathrm{db} / \mathrm{db}$ diabetic mice. Aortas were stained with $\mathrm{H}$ and $\mathrm{E}$ stain (20X). Black arrows indicate adipose tissue and fat deposits; black bars indicate the thickness of the vascular wall; asterisks indicate atheroma. Cardiac tissue sections were examined using Masson's trichrome staining: fibrosis can be recognized by blue staining. The thickness of the vascular wall is different depending on the treatment (40X).

\section{Discussion}

We researched the effect Resveratrol alone and in combination with Pioglitazone had on the cardiovascular complications of $\mathrm{db} / \mathrm{db}$ diabetic mice. Treatments were administered for 6 weeks, and at the end of the study, we evaluated different biochemical clinical parameters. We employed histopathological studies to evaluate the effects on cardiac tissue and thoracic aortas.

Nowadays, cardiomyopathy frequently accompanies Type $2 \mathrm{DM}$ and is responsible for about million deaths each year among DM Type 2 sufferers [21]. The main alterations in cardiomyopathy include hypertrophy and degeneration of cardiomyocytes, augmented cell adhesiveness, decreased fibrinolysis, and alterations in permeability [22]. Hyperglycaemia and insulin resistance are the most important risk factors that cause morphological and functional damage to cardiovascular tissue [23].

Resveratrol is a flavonoid that has been shown to have numerous beneficial actions in Type 2 DM [19]. Recently, several studies have pointed out resveratrol can reduce obesity, hypertriglyceridemia, the atherosclerotic state and cardiovascular dysfunction [24]. The mechanism of action generally improves Type 2 DM symptoms and its cardiovascular complications, mainly via its anti-inflammatory and antioxidant effects [25].

Our research shows that diabetic mice treated with resveratrol alone did not display a modified lipid profile but had improved glucose levels and glycosuria. However, an important change in glucose metabolism and lipid profile was observed when diabetic mice were treated with resveratrol and pioglitazone. Multiple studies have shown the antidiabetic effects of resveratrol, which is associated with an improvement of insulin resistance and secretion, a reduction of $\mathrm{HbAl}$ and even an improvement in HDL levels [26]. On the other hand, it has also been demonstrated that pioglitazone is able to reduce insulin resistance in several sites by binding to the peroxisome Proliferator-Activated Receptor (PPAR)- $\gamma$ [27]. Additionally, pioglitazone improves plasma lipid levels [28]. The improvement of glucose and lipid metabolism in diabetic mice treated with the combined therapy suggests a synergistic effect, and this is because each drug has a different mechanism of action.

Diabetic cardiomyopathy is characterized by the progression of cardiac dysfunction, death cell and tissue remodeling due to the replacement of the myocardium by non-functional fibrotic tissue [23]. It has also been observed that cardiomyoblasts exposed to high concentrations of glucose increase TGF- $\beta$ activity and promote collagen synthesis [29]. Our results showed that diabetic mice treated with resveratrol alone, as well as those treated with resveratrol and pioglitazone, displayed a significant reduction in cardiac fibrosis. It has been pointed out that resveratrol can influence molecular pathways associated with processes involved with cell survival, inhibition of apoptosis, carbohydrate metabolism, and regulation of protein synthesis. It is also able to phosphorylate and activate endothelial nitric oxide synthase, which is important in heart regulation and restoration [30]. Resveratrol has a potential anti-atherosclerotic effect, inhibiting proliferation of fibroblasts and cell apoptosis [31]. Recently, it has been found that resveratrol can modify not only those molecular pathways but also epigenetic machinery. Resveratrol treatment induces the activation of SIRT3 and inhibits the TGF- $\beta / S m a d 3$ pathway in mouse hearts. Specifically, it has been observed to inhibit fibroblast to myoblast conversion and enhance cardiac function while reducing fibrosis in the myocardium [32]. There are no reports about the antifibrotic effect of pioglitazone in the heart. However, it has been found that PPAR- $\gamma$ agonists are able to modify processes as fibrosis and inflammation in the liver and kidney, possibly by inhibiting TGF$\beta 1$ and increasing the expression of morphogenic proteins as potential anti-fibrotic agents [33]. Pioglitazone might have the same effects in the heart and a combined effect with resveratrol can help improve upon cardiac fibrosis.

The Vascular Cell Adhesion Molecule-1 (VCAM-1) is necessary for the anchorage of monocytes and lymphocytes and is highly expressed in the inflamed vascular endothelium [34]. VCAM-1 has a dominant role in the initiation of the atherosclerotic process and can serve as an indicator of endothelial activation, a molecule marker of chronic vascular inflammatory processes, and a prognostic factor of cardiovascular disease. Our current research evidence that resveratrol, whether alone or combined with pioglitazone, considerably decreased the expression of VCAM-1 in the blood vessels and myocardium of diabetic mice. It has been previously reported that resveratrol attenuates vascular endothelial inflammation by activating autophagy [35]. Besides that, several preclinical research studies have revealed that resveratrol has anti-inflammatory effects and reduces inflammatory markers such as aortic macrophage infiltration, ICAM-1 and NFK- $\beta$ expression in atherosclerosis models [36,37]. Pioglitazone was shown to exert a dose-dependent positive action on endothelial cell death inhibition and ameliorate atherosclerosis progression in both carotid and coronary circulation, as well as reduce cardiovascular events [38]. Those effects can explain the improvement in the structure of the thoracic aortas and cardiac vessels observed in diabetic mice treated both with resveratrol alone and in combination with pioglitazone. 


\section{Conclusion}

The current study demonstrated that a combined therapy of resveratrol/pioglitazone improves concentrations of circulating lipids and glucose, as well as ameliorating diabetic cardiomyopathy in diabetic mice by producing an additive pharmacological effect. Present data suggest that the resveratrol/pioglitazone combination optimizes the secretion and use of insulin in the body. Lower circulating insulin, along with lower blood levels of both HbA1c and glucose, might jointly reduce cardiac insulin resistance and therefore lead to a reduced cardiovascular risk. The combined use of resveratrol and pioglitazone has therapeutic potential against diabetic cardiomyopathy.

\section{Acknowledgment}

We wish to thank CONACYT Mexico for its financial support (Project 251925).

\section{References}

1. Harcourt BE, Penfold SA, Forbes JM (2013) Coming full circle in diabetes mellitus: from complications to initiation. Nat Rev Endocrinol 9: 113-23.

2. Miki T, Yuda S, Kouzu H, Miura T (2013) Diabetic cardiomyopathy: pathophysiology and clinical features. Heart Fail Rev 18: 149-166.

3. Chavali V, Tyagi SC, Mishra PK (2013) Predictors and prevention of diabetic cardiomyopathy. Diabetes Metab Syndr Obes Targets Ther 6: 151-160.

4. Kuethe F, Sigusch HH, Bornstein SR, Hilbig K, Kamvissi V, et al. (2007) Apoptosis in patients with dilated cardiomyopathy and diabetes: A feature of diabetic cardiomyopathy? Horm Metab Res 39: 672-676.

5. Bugger H, Abel ED (2014) Molecular mechanisms of diabetic cardiomyopathy. Diabetologia 57: 660-671.

6. Kehat I, Molkentin JD (2010) Molecular pathways underlying cardiac remodeling during pathophysiological stimulation. Circulation 122: 2727-2735.

7. Sivasankar D, George M, Sriram DK (2018) Novel approaches in the treatment of diabetic cardiomyopathy. Biomed Pharmacother 106: 1039-1045.

8. Levelt E, Gulsin G, Neubauer S, McCann GP (2018) Mechanisms in endocrinology: diabetic cardiomyopathy: pathophysiology and potential metabolic interventions state of the art review. Eur J Endocrinol 178 . R127-R139.

9. Qian L, Thapa B, Hong J, Zhang Y, Zhu M, et al. (2018) The present and future role of ultrasound targeted microbubble destruction in preclinical studies of cardiac gene therapy. J Thorac Dis 10: 1099-1111.

10. Alonso N, Moliner P, Mauricio D. Pathogenesis (2018) Clinical features and treatment of diabetic cardiomyopathy. Adv Exp Med Biol 1067: 197-217.

11. Ruiz M, Coderre L, Allen BG, Des Rosiers C (2018) Protecting the heart through MK2 modulation, toward a role in diabetic cardiomyopathyand lipid metabolism. Biochim Biophys Acta Mol Basis Dis 1864: 1914-1922.

12. Staels B (2017) Cardiovascular protection by sodium glucose cotransporter 2 inhibitors: potential mechanism. Am J Cardiol 120: S28S36.

13. Voulgari C, Papadogiannis D, Tentolouris N (2010) Diabetic cardiomyopathy: from the pathophysiology of the cardiac myocytes to current diagnosis and management strategies. Vasc Health Risk Manag 6: 883-903.

14. Flory JH, Ukena JK, Floyd JS (2016) Novel anti-glycemic drugs and reduction of cardiovascular risk in diabetes: Expectations, realized, promises unmet. Curr Atheroscler Rep 18: 79.

15. Liao HW, Saber JL, Wu YL, Chen TH, Lee M, et al. (2017) Pioglitazone and cardiovascular outcome in patients with insulin resistance, prediabetes and Type 2 diabetes: A systemic review and meta-analysis. BMJ e013927.

16. Rauf A, Imran M, Suleria HAR, Ahmad B, Peters DG, et al. (2017) A comprehensive review of the health perspectives of resveratrol. Food Funct 8: 4284-4305.

17. Cho S, Namkoong K, Shin M, Park J, Yang E, et al. (2017) Cardiovascular protective effects and clinical applications of resveratrol. J Med Food 20: 323-334.

18. Vassort G, Turan B (2010) Protective role of antioxidants in diabetesinduced cardiac dysfunction. Cardiovasc Toxicol 10: 73-86.

19. Diaz-Gerevini GT, Repossi G, Dain A, Tarres MC, Das UN, et al. (2016) Beneficial action of resveratrol: How and why? Nutrition 32: 174-178.

20. National Research Council US (2011) Committee for the update of the guide for the care and use of laboratory animals, guide for the care and use of laboratory animals (8th edn.). National Academies Press, Washington DC.

21. Voulgari C, Papadogiannis D, Tentolouris N (2010) Diabetic cardiomyopathy: from the pathophysiology of cardiac myocytes to current diagnosis and management strategies. Vasc Health Risk Ma 6: 883-903.

22. Iikun O, Boudina S (2013) Cardiac dysfunction and oxidative stress in the metabolic syndrome: an update on antioxidant therapies. Curr Pharm Des 19: 4806-4817.

23. Hu X, Bai T, Xu Z, Liu Q, Zheng Y, et al. (2017) Pathophysiological fundamental of diabetic cardiomyopathy. Compr Physiol 7: 693-711.

24. Bonnefont-Rousselot D (2016) Resveratrol and cardiovascular diseases. Nutrients 8: E250.

25. Zordoky BNM, Robertson IM, Dyck JRB (2015) Preclinical and clinical evidence for the role of resveratrol in the treatment of cardiovascular disease. Biochim Biophys Acta 1852: 1155-1177.

26. Xia N, Daiber A, Forstermann ULH (2017) Antioxidant effect of resveratrol in the cardiovascular system. Br J Pharm 174: 1633-1646.

27. Friedland SN, Leong A, Filion KB, Genest J, Lega IC, et al. (2012) The cardiovascular effects of peroxisome proliferator active receptor agonists. Ame J Med 125: 126-133.

28. Betteridge J (2007) Effects of pioglitazone on lipid and lipoprotein metabolism. Diabetes Obes Metab 9: 640-647.

29. Mandavia CH, Aroor AR, DeMarco VG, Sowers JR (2013) Molecular and metabolic mechanisms of cardiac dysfunction in diabetes. Life Sci 92: 601-608.

30. Palfi A, Bartha E, Copf L, Marl L, Gallyas F, et al. (2009) Alcohol-free red wine inhibits isoproterenol-induced cardiac remodeling in rats by the regulation of Akt1 and protein kinasa $C \alpha / \beta$ II. J Nutr Biochem 20: 418-425.

31. Chen T, Li J, Liu J, Li N, Wang S, et al. (2015) Activation of SIRT3 by resveratrol ameliorates cardiac fibrosis and improves cardiac function via the TGF- $\beta /$ Smad3 pathway. Am J Physiol 308: H424-434.

32. Grimaldi V, De Pascale MR, Zullo A, Soricelli A, Infante T, et al. (2017) Evidence of epigenetic tags in cardiac fibrosis. J Cardiol 69: 401-408.

33. Nagao S, Yamaguchi T (2012) PPAR- $\gamma$ agonists in polycystic kidney disease with frequent development of cardiovascular disorders. Curr Mol Pharm 5: 292-300.

34. Derosa G, Maffioli P (2016) A review about biomarkers for the investigation of vascular function and impairment in diabetes mellitus. Vasc Health Risk Manag 12: 415-419.

35. Huang FC, Kuo HC, Huang YH, Yu HR, Li SC, et al. (2017) Antiinflammatory effect of resveratrol in human coronary arterial endothelial cells via induction of autophagy: implications for the treatment of Kawasaki disease. BMC Pharmacol Toxicol 18: 3-11.

36. Coutinho DS, Pacheco MT, Frozza RL, Bernanrdi A (2018) Antiinflammatory effects of resveratrol: mechanistic insights. Int J Mol Sci 19: 181.

37. Radenkovic M (2014) Pioglitazone and endothelial dysfunction: Pleiotropic effects and possible therapeutic implications. Sci Pharm 82: 709-721. 
Citation: Duarte-Vazquez MA, Solis AG, Esparza JR, Rosado JL, Fragoso LR (2018) Resveratrol Combined with Pioglitazone Ameliorates Cardiovascular Complications in db/db Diabetic Mice. J Metabolic Synd 7: 243. doi:10.4172/2167-0943.1000243

Page 7 of 7

38. Jialal I, Devaraj S (2015) Anti-inflammatory strategies to prevent diabetic cardiovascular disease. Clin Pharmacol Ther 98: 121-123. 